\title{
Determinants of Density- and Frequency-Dependent Fitness in Competing Plant Pathogens
}

\author{
M. R. Newton, L. L. Kinkel, and K. J. Leonard
}

First, second, and third authors: Department of Plant Pathology, University of Minnesota; and third author: USDA, ARS Cereal Rust Laboratory, University of Minnesota.

Accepted for publication 28 September 1997.

\begin{abstract}
Newton, M. R., Kinkel, L. L., and Leonard, K. J. 1998. Determinants of density- and frequency-dependent fitness in competing plant pathogens. Phytopathology 88:45-51.

Using mathematical models, we investigated how infection and sporulation characteristics of competing plant pathogens determine the density and frequency dependence of relative fitness. Two models, one for the infection stage and one for the sporulation stage of a pathogen's life cycle, describe reproductive output of pathogen strains in mixture as a function of the strains' population densities. Model parameters include infection and

life cycle stages. In this work, paired hypothetical strains were assigned equal baseline parameter values. Parameters were then altered one at a time for one or both strains, and relative fitness was calculated over a range of densities and strain frequencies. Except for infection efficiency, the fitness benefit conferred by an advantage in a single parameter was always density dependent. Relative fitness was frequency dependent whenever inter- and intrastrain competitive effects were not equal. These results suggest that the fitness of pathogens in nature is rarely fixed, but, rather, may typically be highly dependent on the densities and frequencies of all coexisting strains in a habitat.
\end{abstract} sporulation efficiencies, carrying capacities on leaves for sporulating lesions and spore production, and coefficients of interstrain competitive effects in both life cycle stages. Although the models were originally developed for rust fungi, they are generally applicable to any organism with distinct colonization (e.g., infection) and propagative (e.g., sporulation)
Additional keywords: competition model and effects, foliar fungi, infection and sporulation life cycle stages, reproductive versus interactive traits, $r$ and $K$ selection.
To understand the population dynamics of coexisting organisms, it is necessary to analyze the manner in which their reproductive capacities and competitive abilities influence relative fitness (relative per-individual contribution to the next generation's gene pool) (29) in mixtures. While interorganismal competitive ability, or the ability to inhibit another organism while resisting inhibition by that organism, is an important component of relative fitness, it is not the sole determinant of fitness. Relative fitness in mixture is also influenced by noninteractive reproductive characteristics such as carrying capacities or basic reproductive rates that can be measured in the absence of a specific competitor in a given environment. The failure to consistently separate the concepts of competitive ability and fitness in much of the basic and applied research (10, $13,27,28,32,35,36)$ can create confusion about the significance of organismal interactions within a population or community. In studying the relative success of coexisting organisms, it is, therefore, important to develop methods that distinguish the specific contributions of differences in competitive abilities from differences in intrinsic reproductive abilities of organisms to their relative fitness in mixture.

Fitness of organisms may depend on the absolute population densities of all organisms present or on both the total density and relative frequencies of the organisms. The topics of density- and frequency-dependent selection, or the dependence of the fitness of a genotype (or allele) on its density or frequency within a population (8), have aroused much interest in ecology and population genetics $(1-3,5,21,34)$. The attention given to these topics stems from a

Corresponding author: M. Newton; E-mail address: miriamn@puccini.crl.umn.edu

Publication no. P-1997-1106-01R

This article is in the public domain and not copyrightable. It may be freely reprinted with customary crediting of the source. The American Phytopathological Society, 1998. growing realization that an organism's fitness is not necessarily an inherent, fixed property, but may be appreciably influenced by intraand interspecific density effects, and that density- or frequencydependent selection may help explain the maintenance of diverse genotypes within populations. Theoretical models based on LotkaVolterra competition equations $(22,33)$ have been used to quantitatively describe the dependence of population dynamics in binary mixtures on the absolute densities of both organisms in some systems (1). Although there is evidence in the microbial literature for density effects on per-individual reproductive output $(6,12,15,18$, $27,30,31,37)$, these studies have generally dealt with density effects on reproductive output in single strains or species rather than the effects of densities of coexisting organisms on relative fitness in mixtures. The development and application of models quantifying the influences of population densities and frequencies on microbial fitness in mixed populations will enhance our ability to understand and predict the efficacy of biological control agents, the progress of disease epidemics, and long-term changes in the prevalence of microbial strains or species.

We developed models to describe reproductive dynamics in coexisting strains of a fungal plant pathogen, Puccinia graminis $\mathrm{f}$. sp. tritici. Reproductive output for two coexisting strains was modeled as a function of the strains' population densities for two successive life cycle stages: the infection stage (in which spores infect the plant surface and form lesions) and the sporulation stage (in which spores are produced in lesions). The models for the two life cycle stages are similar functions with analogous parameters measuring traits that contribute to fitness. The models measure both reproductive and interactive traits. Reproductive traits include reproductive efficiencies (infection efficiency of spores or sporulation efficiency of lesions) and carrying capacities on leaves (for formation of lesions or spores), and interactive traits include interstrain competitive effects for lesion or spore production. Model parameters are density independent and can, therefore, be quantified 
and their effects on fitness predicted over a broad range of population densities and strain frequencies. Although the models were specifically derived for rust fungi, fitness traits similar to the reproductive efficiency and carrying capacity parameters in the infection and sporulation models are also characteristic of a wide variety of other organisms.

The goals of this study were to use the models to investigate the contribution of each of the defined fitness traits to a strain's overall fitness and to explore the ways in which these contributions vary with the density and frequency of the strains. A summary of a portion of these results has been published in an abstract (24).

\section{MATERIALS AND METHODS}

We developed two models describing interactions between strains of $P$. graminis f. sp. tritici on wheat leaves for two successive pathogen life cycle stages. The models are presented in detail elsewhere (25); here, an overview is presented. Each model describes the impact of competitive interactions on the relationship between population density and reproductive output at a distinct stage of the asexual cycle. The "infection model," based on the multiple infection transformation (9), was developed to describe the relationship between numbers of urediniospores of each $P$. graminis $\mathrm{f}$. sp. tritici strain inoculated onto the leaf and the number of sporulating lesions (uredinia) produced by each strain on the leaf. The assumptions of this model follow the widely accepted argument of the multiple infection transformation (9): the strains compete on a localized scale for individual infection sites on leaves, or for resources within those sites; and no more than one uredinium forms at any single infection site. We also assume that no single uredinium produces urediniospores of more than one strain (M. R. Newton and K. J. Leonard, personal communication; from repeated inoculations with mixtures of genetically marked strains of $P$. graminis f. sp. tritici).

The "sporulation model" was developed to describe the relationship between numbers of uredinia of both strains on a leaf and the numbers of urediniospores produced by each strain. This model is based on the monomolecular equation (4). Unlike competition for uredinial formation, competition among uredinia for spore production is formulated to occur across the entire leaf rather than in localized sites on the leaf. This is because uredinia establish metabolic sinks that induce the host leaf to translocate nutrients from other parts of the leaf to the uredinial site $(7,11,26)$.

Experimental data reveal that saturation patterns occur for increases in reproductive output (uredinia or urediniospores) relative to increasing population density (inoculated urediniospores or formed uredinia) and that the saturation plateaus (carrying capacities) differ for different strains in both reproductive phases of $P$. graminis f. sp. tritici (25). The use of two different models was not suggested by obvious differences in data patterns for the two reproductive phases, but was based upon conceptual biological assumptions about the way in which $P$. graminis f. sp. tritici strains compete with each other in the different phases (local versus generalized competition). Both models are generally applicable to a variety of competitive interactions in different microbial systems such as plant epiphytic bacteria (17; K. Stromberg, unpublished data).

Infection model: Relationship between inoculum density and uredinia per leaf. First, we considered the case in which one strain is inoculated onto a leaf in the absence of another strain. We assumed that (i) urediniospores are distributed randomly over the leaf during inoculation; (ii) prior to inoculation, all of the infection sites on a leaf are equally likely to become infected by a given urediniospore; and (iii) all of the urediniospores of a strain have the same likelihood of forming an infection. $E$ is the infection efficiency of a strain or the probability that a urediniospore inoculated onto a leaf will infect it, and $K$ is the finite number of micro- sites on a leaf where uredinia of a strain may develop. Multiple spores may infect a site and produce mycelial colonies, but only one colony originating from one spore will survive and grow into a mature, sporulating uredinium within each infected site.

The probability that an inoculated urediniospore will infect a leaf in a particular site is $E / K$. From the binomial distribution (14), the probability that no urediniospores will infect a particular site is $[1-(E / K)]^{S}$, in which $S$ is the number of urediniospores inoculated onto the leaf. The probability that a site will develop a uredinium is equivalent to the probability that a site will have at least one infection, which equals $1-[1-(E / K)]^{S}$. Therefore, the number of uredinia, $U$, that will develop per leaf following a single-strain inoculation is described by the formula

$$
U=K\left\{1-[1-(E / K)]^{S}\right\}
$$

Next we considered two strains, strain 1 and strain 2 , with potentially different carrying capacities and infection efficiencies. $x_{1}$ and $x_{2}$ represent the numbers of infections of strains 1 and 2 in an infection site. The proportion of infections of strain 1 in the site will be $x_{1} /\left(x_{1}+x_{2}\right)$, which also equals the probability that a strain 1 uredinium will develop in that site if the two strains are equally competitive. If the two strains are not equally competitive, $\alpha_{2}$ is a competition coefficient measuring the effect of an infection of strain 2 on the probability that an individual strain 1 infection in the site will develop into a uredinium relative to the effect of another infection of strain 1 on the chances of that strain 1 infection winning the site. In that case, the probability that a strain 1 infection will outcompete the other infections and develop into a uredinium in the site equals $x_{1} /\left(x_{1}+\alpha_{2} x_{2}\right)$. A coefficient value less than 1 indicates that an individual infection of strain 2 has a weaker inhibitory (e.g., crowding) effect on the development of strain 1 infections into uredinia than individual strain 1 infections have upon each other, whereas a value greater than 1 indicates that strain 2 infections inhibit the development of uredinia by strain 1 infections more strongly than strain 1 infections inhibit each other.

As the number of individuals in the population (i.e., population density) increases, the total impact of competition on any specific organism in the population will increase, because it will be interacting with more individuals. In a mixed population, if strain 2 has a competition coefficient of 2.0, the crowding impact of each strain 2 individual on strain 1 will be equivalent to that of two strain 1 individuals on strain 1. In our model, this ratio of relative impacts of each strain on the other is constant over density. This means that individuals of strain 2 do not become progressively more inhibitory to strain 1 as population density increases. Rather, the total impact of competition on a population is a function of the number of individuals present adjusted according to the competition coefficients of each strain. Our tests of residuals from regression from previous experimental work with $P$. graminis f. sp. tritici (25) revealed no systematic error in our assumption of competition coefficients being constant over population density.

The expected proportion of infection sites for strain 1 in which strain 1 uredinia will form, $\operatorname{EXP}\{\operatorname{pr} 1\}$, is the expected probability that a site will be infected by strain 1 and that a strain 1 uredinium will establish in that site. This is equal to the expected value of $x_{1} /\left(x_{1}+\alpha_{2} x_{2}\right)$ over the distribution of all possible combinations of infections of the two strains in a site that could result in the formation of a strain 1 uredinium ( $x_{1}$ can range from 1 to $S_{1} ; x_{2}$ can range from 0 to $S_{2}$ ). This expectation can be calculated as the summation over all possible values of $x_{1}$ and $x_{2}$ of $P\left(x_{1}\right) P\left(x_{2}\right) x_{1} /\left(x_{1}\right.$ $\left.+\alpha_{2} x_{2}\right)$, in which $P\left(x_{i}\right)$ is a binomial $\left(S_{i}, E_{i} / K_{1}\right)$ probability. This summation can be approximated to a high degree of accuracy by summing over a reasonable subset of possible $x_{1}$ and $x_{2}$ values. In this study, the expected proportion was approximated by considering $x_{1}$ and $x_{2}$ from $0\left(x_{2}\right)$ or $1\left(x_{1}\right)$ to 50. Higher values resulted in no further changes in predicted numbers of uredinia to the thou- 
sandths decimal place in all cases (data not shown). Therefore, the expected number of uredinia of strain 1 that will form is

$$
U_{1}=K_{1}(\operatorname{EXP}\{\operatorname{pr} 1\})
$$

Sporulation model: Relationship between uredinia per leaf and urediniospores produced per leaf. Again, first we considered the case in which only one strain is present on a leaf. We assumed that leaves have limited resources that uredinia can draw upon for sporulation and, therefore, there is a finite maximum number of urediniospores that can be produced on an infected leaf. We assumed also that, when the number of urediniospores produced is near the maximum, fewer resources are available to each uredinium for sporulation and, thus, fewer urediniospores are produced per uredinium. Stated precisely, we assumed that, on leaves infected by a single strain, the rate of change of urediniospore production per leaf with respect to increasing numbers of uredinia is proportional to the difference between maximum and actual levels of urediniospore production: $d Q / d U=D(M-Q)$, in which $Q$ is the number of urediniospores produced per leaf, $U$ is the number of uredinia per leaf, $M$ is the maximum sporulation capacity (maximum number of urediniospores that can be produced per leaf), and $D$ is a rate parameter relative to sporulation efficiency (determines the proportional rate of approach of urediniospores produced per leaf to the maximum sporulation capacity with respect to increasing uredinial numbers). Solving the differential equation yields $Q=M-C e^{-D U}$, in which $C$ is a constant of integration. However, since $Q=0$ when $U=0, C=M$; therefore, $Q=M\left(1-e^{-D U}\right)$.

It should be noted that $D$ provides an indication of sporulation efficiency specifically relative to $M$, since it describes the rate of approach of urediniospore production to the maximum capacity with respect to uredinial density. To obtain an absolute measure of sporulation efficiency that is not linked with the maximum sporulation capacity, $A$ is the number of spores a single uredinium can produce in the absence of any other uredinia on the leaf. The parameter $A$ is the value of $Q$ when $U=1: A=M\left(1-e^{-D}\right)$. Solving for $D$ yields $D=-\ln (1-A / M)$. Substitution of this quantity for $D$ in the equation results in the reparameterization

$$
Q=M\left\{1-[1-(A / M)]^{U}\right\}
$$

This turns out to be exactly analogous to the function that describes the single-strain relationship between $S$ and $U$, illustrating the similarity between the two parts of the model. Thus, $A$ is a sporulation efficiency parameter analogous to $E$, the infection efficiency parameter.

On leaves infected with both strains 1 and 2, the competitive effect of strain 2 on urediniospore production by strain 1 is measured by the competition coefficient, $\beta_{2}$, which measures the effect of a uredinium of strain 2 on urediniospore production per uredinium in strain 1 relative to the effect of a uredinium of strain 1 on urediniospore production per uredinium in strain 1. Coefficient values of less than 1 signify that the interstrain competitive effect of a strain 2 uredinium on strain 1 sporulation per uredinium is not as strong as the intrastrain effect of a strain 1 uredinium upon strain 1 sporulation per uredinium and, likewise, coefficient values greater than 1 signify that strain 2 inhibits strain 1 sporulation more strongly than strain 1 inhibits its own sporulation. The coefficient can be thought of as a means to convert $U_{2}$ into the same "units" of inhibitory effect as uredinia of strain 1, so that the "effective" total number of uredinia per leaf as perceived by strain 1 is $U_{1}+\beta_{2} U_{2}$ and, thus, the effective proportion of strain 1 uredinia in the mixture is $U_{1} /\left(U_{1}+\beta_{2} U_{2}\right)$. The maximum number of strain 1 urediniospores that can be produced on a leaf infected with both strains will equal the maximum sporulation capacity for strain 1 multiplied by the effective proportion of strain 1 uredinia: $M_{1} U_{1} /\left(U_{1}+\beta_{2} U_{2}\right)$. Therefore, substituting this value for $M$ in the single-strain equation provides a function to describe the number of strain 1 urediniospores produced on leaves infected by strains 1 and 2. Finally, replacing $U$ in the single-strain equation with the effective total number of uredinia in a mixed inoculation, the equation relating strain 1 urediniospore production to the uredinial densities of both strains on the leaf is

$$
Q_{1}=\left(\frac{U_{1}}{U_{1}+\beta_{2} U_{2}}\right) M_{1}\left\{1-\left[1-\left(A_{1} / M_{1}\right)\right]^{\left(U_{1}+\beta_{2} U_{2}\right)}\right\}
$$

Combining infection and sporulation models: Relationship between inoculum density and urediniospores produced per leaf. By combining the equations for uredinia as a function of urediniospores inoculated and sporulation as a function of uredinia, the number of urediniospores produced by a strain on a leaf can be predicted as a function of the numbers of urediniospores of the two strains inoculated onto the leaf.

Model calculations. Model calculations were performed using SAS statistical software (SAS Institute, Inc., Cary, NC). Parameter values used in the calculations were chosen to be similar to estimated values obtained in previous experimental work (25). Each strain was assigned the baseline parameter values $E=5.0 \times$ $10^{-2}, A=4.0 \times 10^{3}, K=4.0 \times 10^{2}, M=2.0 \times 10^{5}, \alpha=1$, and $\beta=1$. The value of one parameter at a time was then altered in one or both strains. First, we investigated the cases in which strain 1 had a 50\% advantage in each parameter. Other cases investigated included situations in which all parameters were assigned baseline values except $\left(\alpha_{1}=1, \alpha_{2}=0.67\right),\left(\beta_{1}=1, \beta_{2}=0.67\right),\left(\beta_{1}=\beta_{2}=\right.$ $1.5)$, or $\left(\beta_{1}=\beta_{2}=0.67\right)$. For the last two cases, the analogous combinations for the infection model, $\left(\alpha_{1}=\alpha_{2}=1.5\right)$ and $\left(\alpha_{1}=\alpha_{2}\right.$ $=0.67$ ), were not investigated, since it was computationally more intensive to obtain results for different values of $\alpha$ and relative fitness results indicate that specific combinations of competition coefficients produce qualitatively similar results for the two models.

For all of these cases, model predictions of urediniospore production were made at 14 different total inoculum densities (ranging from 100 to 100,000 urediniospores per leaf) and at seven different frequencies of the two strains in the inoculum (proportion of strain 1 urediniospores in inoculum $=0,0.1,0.3,0.5,0.7,0.9$, and 1). The initial inoculum densities and final reproductive outputs of the two strains were used to calculate the relative fitness of strain 1 at each combination of density, frequency, and parameter values. Relative fitness was calculated as defined by Leonard (19): the fitness of strain 1 relative to strain 2, which we will denote $w_{1}$, equals

$$
\frac{\left(\frac{Q_{1}}{Q_{1}+Q_{2}}\right) /\left(\frac{S_{1}}{S_{1}+S_{2}}\right)}{\left(\frac{Q_{2}}{Q_{1}+Q_{2}}\right) /\left(\frac{S_{2}}{S_{1}+S_{2}}\right)}
$$

which simplifies to $S_{2} Q_{1} / S_{1} Q_{2}$. This quantity can be thought of as spore production per spore inoculated of strain 1 relative to strain 2. For each parameter combination, relative fitness values were evaluated to the hundredths decimal place. Since the models are deterministic rather than stochastic, any changes in relative fitness with density or frequency were considered evidence for densityor frequency-dependent fitness of coexisting pathogen strains.

\section{RESULTS AND DISCUSSION}

Using our models, we evaluated the reproductive dynamics of coexisting microbial strains on leaves under conditions in which strains differed in none or only one of the following traits: reproductive efficiency ( $E$, infection efficiency, or $A$, sporulation efficiency), carrying capacity ( $K$, uredinial carrying capacity, or $M$, sporulation carrying capacity), and the competitive effect of a strain on the other strain's reproductive output $(\alpha$, competitive effect on the other's uredinial formation, or $\beta$, competitive effect on the other's urediniospore production). For the simplest (null) case, when 
corresponding parameter values were equal and competition coefficients were equal to 1 (interstrain competition = intrastrain competition) for both strains, neither strain had an advantage in relative fitness $\left(w_{1}=1.0\right)$, regardless of the density or frequency of the strains. Therefore, any trend observed in relative fitness as a result of varying a single parameter was unambiguously due to the individual effect of that one parameter.

Advantages in a single parameter in one strain often conferred a density- or frequency-dependent selective advantage to that strain (Table 1). Even though the two models have similar formulations for single-strain population dynamics, the analogous parameters in the two life stages do not affect fitness in mixed populations in the same way. For example, a $50 \%$ advantage in infection efficiency $(E)$ conferred a fitness advantage of essentially $50 \%\left(w_{1} \approx 1.5\right)$ at all population densities and frequencies tested (e.g., at $S=100, w_{1}=$

TABLE 1. Effects of population density and genotype frequency on the contributions of specific traits to relative fitness

\begin{tabular}{|c|c|c|c|c|}
\hline \multirow[b]{2}{*}{ Parameter type } & \multirow[b]{2}{*}{ Model } & \multirow{2}{*}{$\begin{array}{c}\text { Parameter } \\
\text { combination }^{\mathrm{a}}\end{array}$} & \multicolumn{2}{|c|}{ Dependence } \\
\hline & & & Density ${ }^{b}$ & Frequency $^{c}$ \\
\hline & & $\begin{array}{l}\text { Equal baseline values } \\
\text { for both strains }\end{array}$ & No & No \\
\hline $\begin{array}{l}\text { Reproductive } \\
\text { efficiency }\end{array}$ & $\begin{array}{l}\text { Infection } \\
\text { Sporulation }\end{array}$ & $\begin{array}{l}E_{1} / E_{2}=1.5 \\
A_{1} / A_{2}=1.5\end{array}$ & $\begin{array}{l}\text { No } \\
\text { Yes (-) }\end{array}$ & $\begin{array}{l}\text { No } \\
\text { No }\end{array}$ \\
\hline $\begin{array}{l}\text { Carrying } \\
\text { capacity }\end{array}$ & $\begin{array}{l}\text { Infection } \\
\text { Sporulation }\end{array}$ & $\begin{array}{l}K_{1} / K_{2}=1.5 \\
M_{1} / M_{2}=1.5\end{array}$ & $\begin{array}{l}\text { Yes }(+) \\
\text { Yes }(+)\end{array}$ & $\begin{array}{l}\text { No } \\
\text { No }\end{array}$ \\
\hline $\begin{array}{l}\text { Competitive } \\
\text { ability }\end{array}$ & $\begin{array}{l}\text { Infection } \\
\text { Sporulation }\end{array}$ & $\begin{aligned} \alpha_{1} & =1.5 ; \alpha_{2}=1 \\
\alpha_{1} & =1 ; \alpha_{2}=0.667 \\
\beta_{1} & =1.5 ; \beta_{2}=1 \\
\beta_{1} & =1 ; \beta_{2}=0.667 \\
\beta_{1} & =\beta_{2}=1.5 \\
S_{1} & <S_{2} \\
S_{1} & >S_{2} \\
\beta_{1} & =\beta_{2}=0.667 \\
S_{1} & <S_{2} \\
S_{1} & >S_{2}\end{aligned}$ & $\begin{array}{l}\text { Yes (+) } \\
\text { Yes (+) } \\
\text { Yes (+) } \\
\text { Yes (+) } \\
\text { Yes (-) } \\
\text { Yes (+) } \\
\text { Yes (+) } \\
\text { Yes (-) }\end{array}$ & $\begin{array}{l}\text { Yes (+) } \\
\text { Yes (-) } \\
\text { Yes (+) } \\
\text { Yes (-) } \\
\text { Yes (+) } \\
\text { Yes (+) } \\
\text { Yes (-) } \\
\text { Yes (-) }\end{array}$ \\
\hline
\end{tabular}

${ }^{a}$ With the exception of the particular parameters listed in this column, both strains had equal baseline parameter values (details in text).

b The symbols + or - denote an increase or decrease, respectively, in the relative fitness of strain 1 as total inoculum density increases. For the cases in which $\beta_{1}=\beta_{2} \neq 1$, the direction in which the relative fitness of strain 1 changes with increasing density depends on whether its proportion in the inoculum is less $\left(S_{1}<S_{2}\right)$ or greater $\left(S_{1}>S_{2}\right)$ than that of strain 2 .

c The symbols + or - denote an increase or decrease, respectively, in the relative fitness of strain 1 as the proportion of strain 1 in the inoculum increases.
1.50005, and, at $\left.S=100,000, w_{1}=1.50001\right)$. Preliminary evidence (M. R. Newton, L. L. Kinkel, and K. J. Leonard, unpublished data) indicates that, for values of $E$ much greater than those observed with $P$. graminis $\mathrm{f}$. sp. tritici, the fitness advantage resulting from a $50 \%$ advantage in $E$ may be noticeably greater than $50 \%$ at low population densities, but no different from $50 \%$ at high densities. In contrast, a 50\% advantage in $A$, the analogous parameter in the sporulation model, conferred a fitness advantage close to 1.5 at the lowest densities, but the advantage quickly disappeared ( $w_{1}$ approached 1.0) with increasing population density (Fig. 1A). As with $E$, the fitness advantage associated with an advantage in $A$ was independent of strain frequency.

The difference between the two life stages in the density dependence of fitness when strains differ in reproductive efficiency can be understood by examining the two models. In the infection model, following the usual assumptions of the multiple infection transformation, multiple infections may occur within sites that can support only one uredinium per site. The probability that an infection of a given strain will survive to become a mature uredinium is determined by the expected effective proportion of infections per site $\left[x_{1} /\left(x_{1}+\alpha_{2} x_{2}\right)\right]$, which determines fitness in mixture. This proportion of infections depends on the infection efficiencies of the strains; since an advantage in infection efficiency results in an increased proportion of infections at low and high densities alike, a greater infection efficiency confers a fitness benefit at high as well as low densities. In the sporulation model, on the other hand, uredinia on the leaf are formulated to compete for spore production. At low densities, fitness in mixture is influenced by the sporulation efficiency of the uredinia, but at high densities (when maximum sporulation capacities are equal), fitness is determined primarily by the effective proportion of uredinia $\left[U_{1} /\left(U_{1}+\beta_{2} U_{2}\right)\right]$. Since uredinial proportions are determined prior to sporulation, sporulation efficiency has little effect on fitness at high densities, although superior sporulation efficiency confers a distinct fitness benefit at low densities. These differences illustrate that understanding the biological stage at which interactions occur, i.e., what biological units actually compete with one another, is critical to determining the potential for density dependence of relative fitness.

In contrast to the reproductive efficiency traits, fitness responses to differing carrying capacities were qualitatively similar for the two life stages. A 50\% advantage in $K$ or $M$ conferred negligible fitness advantage at the lowest population densities, but the fitness advantage increased asymptotically to 1.5 with increasing density (Fig. $1 \mathrm{~B}$ and $\mathrm{C}$ ), though the rate of approach to the asymptotic value was much greater for an advantage in $M$ than for an advantage in $K$.

The fact that greater sporulation efficiency increased relative fitness mainly at low population densities, whereas an advantage in

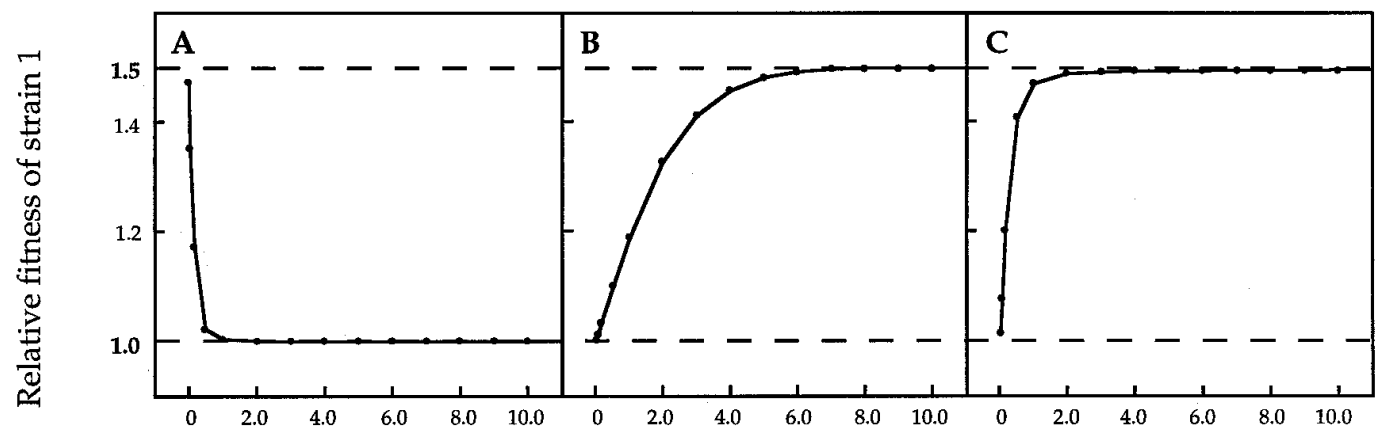

Total population density ( $\times 10^{4}$ urediniospores)

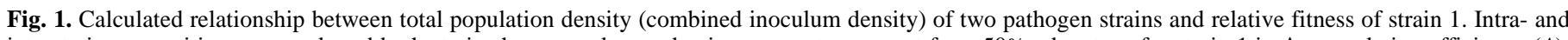

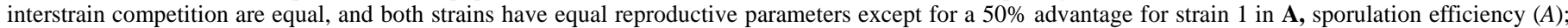
$\mathbf{B}$, uredinial carrying capacity $(K)$; or $\mathbf{C}$, maximum sporulation capacity $(M)$. 
other traits increased relative fitness mainly at high densities, has interesting implications. For two strains having advantages in different traits, population density may determine the direction of selection. These results can be discussed in the language of selection for intrinsic population growth rate and carrying capacity ( $r$ and $K$ selection in ecological literature) (23). High sporulation efficiency represents an $r$-selected trait in that selection would favor the evolution of high sporulation efficiency only at low densities, while increased carrying capacities and competitive effects behave as $K$ selected traits in that selection for high values of these traits would be strongest at high densities. Infection efficiency, which intuitively might seem to be an $r$-selected trait, did not fit neatly into either the $r$ - or $K$-selected category in these calculations, since an advantage in this trait conferred a fitness advantage over a large range of realistically probable densities. These findings may help explain Katsuya and Green's (16) observation of densitydependent fitness of two coinoculated races of $P$. graminis $\mathrm{f}$. sp. tritici in which one race predominated at lower total density, whereas the other predominated at higher density. As indicated by our results, the reversal of fitness advantage of the two races at different population densities could be explained by differences in infection efficiency, sporulation efficiency, carrying capacity for uredinia, and maximum sporulation capacity of the two races, alone or in combination, as well as differences in competition coefficients.

When either or both of the competition coefficients for the two strains had values different from 1 , fitness was both density and frequency dependent. When a strain had a 50\% advantage in its competitive effect on the other strain's infection $(\alpha)$ or sporulation $(\beta)$, the fitness advantage was negligible at the lowest population densities but increased asymptotically with increasing density (Figs. 2 and 3). When $\alpha_{1}=1.5$ and $\alpha_{2}=1.0\left(\alpha_{1} / \alpha_{2}=1.5\right.$, and interstrain competition $\geq$ intrastrain competition for both strains), the fitness advantage of strain 1 was negligible at the lowest frequencies of strain 1 , but increased steadily with increasing frequency of strain 1 (Fig. 2A). However, when $\alpha_{1}=1.0$ and $\alpha_{2}=0.67\left(\alpha_{1} / \alpha_{2}=1.5\right.$, but interstrain competition $\leq$ intrastrain competition for both strains), the fitness advantage of strain 1 was negligible at the highest frequencies of strain 1, but increased steadily as the frequency of strain 1 decreased (Fig. 2B). The same reversal in the direc- tion of frequency dependence of fitness occurred for similar values of $\beta_{1}$ and $\beta_{2}$ (Fig. 3A and B). Thus, the way in which fitness depends on frequency is not solely determined by which strain has the competitive advantage, but on the specific competitive effects (competition coefficient values) the strains have upon one another.

When $\beta_{1}=\beta_{2}>1.0$ (interstrain competition > intrastrain competition for both strains) and all other parameters were equal for strain 1 and strain 2, the fitness of strain 1 increased from less than 1.0 at the lowest frequencies of strain 1 to greater than 1.0 at high frequencies, although this effect was hardly noticeable at the lowest population densities (Fig. 4A). In this case, the strain with the initial advantage in frequency would have the higher relative fitness and eventually replace the other strain in the mixture. When $\beta_{1}=\beta_{2}<1.0$ (interstrain competition < intrastrain competition for both strains) and all other parameters were equal, the fitness of strain 1 decreased from greater than 1.0 at the highest frequencies of strain 1 to less than 1.0 at low frequencies (Fig. 4B). In this case, selection would bring the strain frequencies to equilibrium at $50 \%$, because each strain would have a fitness disadvantage when its frequency rose above $50 \%$. These results indicate that a difference between strains in their frequencies alone can result in a selective advantage of one strain over another even when the strains are completely reproductively and competitively equivalent.

Our results on frequency dependence of fitness confirm the findings of others (2) that frequency-dependent selection can arise solely from competitive interactions among coexisting genotypes within a species. In our predictions for coexisting strains, frequency-dependent selection always occurred except in the special situation in which intra- and interstrain competition are exactly equal for both strains, which is unlikely to happen in nature. This finding concurs with the opinion of Levin (20), who concluded from experience in bacterial research that it would probably be difficult "to come up with examples of 'absolute' (frequency-independent) selection." Our results for the direction of selection with respect to frequency are generally consistent with theoretical expectations (Figs. 2 to 4 and additional parameter combinations not shown). In cases in which $\alpha_{1} \times \alpha_{2}>1$ or $\beta_{1} \times \beta_{2}>1$, selection was positively frequency dependent (i.e., the fitness of a strain increased with increasing
$\mathbf{A}$

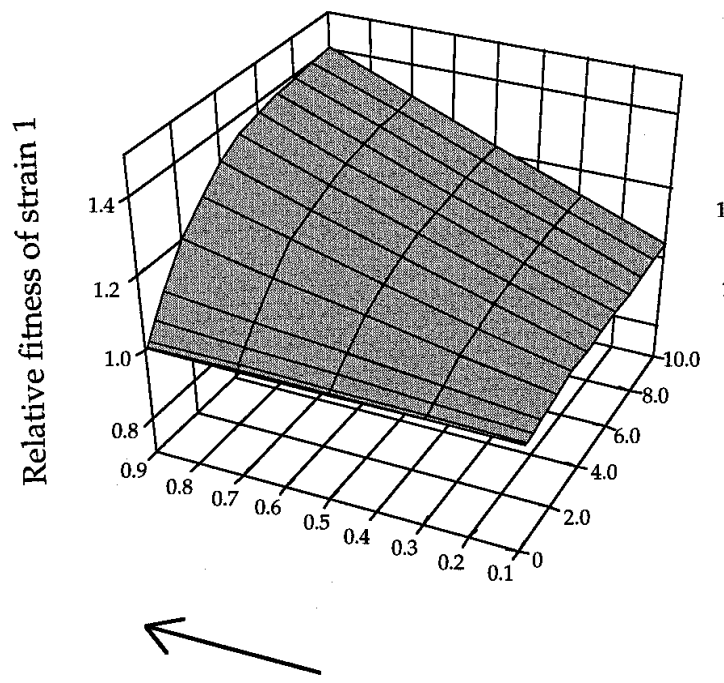

Frequency of strain 1

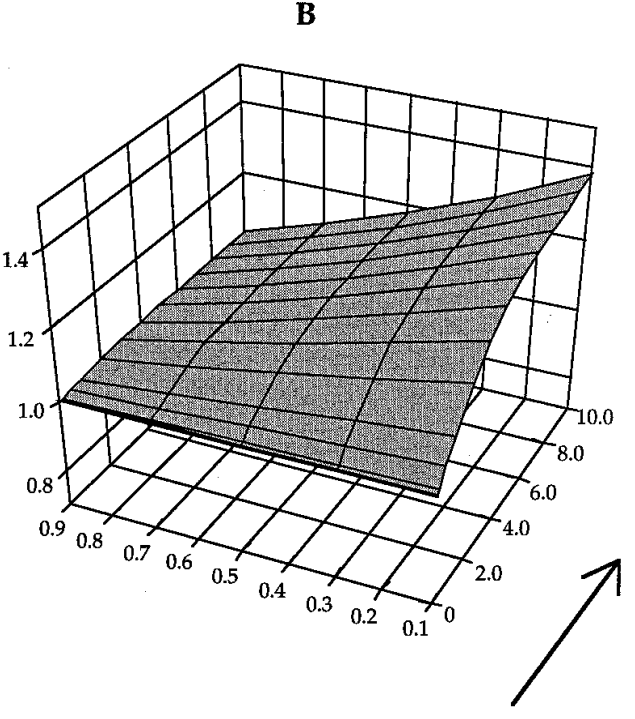

Total population density (x10 ${ }^{4}$ urediniospores)

Fig. 2. Calculated relationships among total population density (combined inoculum density) of two pathogen strains, frequency of strain 1 , and relative fitness of strain 1 when strains differ in their competitive ability for uredinial formation. $\mathbf{A}, \alpha_{1}=1.5$ and $\alpha_{2}=1.0$; and $\mathbf{B}, \alpha_{1}=1.0$ and $\alpha_{2}=0.67$. Note that inter- and intrastrain competition effects are equal when $\alpha=1.0$. Text has details of the calculations. 
frequency of that strain), and, in cases in which $\alpha_{1} \times \alpha_{2}<1$ or $\beta_{1} \times$ $\beta_{2}<1$, selection was negatively frequency dependent (fitness of a strain decreased as its frequency increased), as predicted by classical competition theory (2). Investigation of further parameter combinations may help to strengthen connections between our work and classical theory.

In this study, we have demonstrated how simple combinations of ecological traits in coexisting pathogen strains can result in substantial differences in fitness, even in the absence of differences in competitive ability. Thus, the most fit strain may not be the best competitor, and, conversely, the best competitor may not be the most fit strain. We have also shown that such fitness differences are more likely than not to be highly dependent on strain population densities and frequencies. The way in which this occurs for particular trait combinations provides insight into the roles of those traits in the long-term coexistence or replacement of pathogen strains over time. Although the models used in this study were originally developed for rust fungi, they are generalizable to any system of coexisting organisms that undergo colonization (e.g., infection) and propagative (e.g., sporulation) stages in their life cycles. They provide an important means for exploring the origins of fitness differences in coexisting organisms and for predicting the impacts of specific trait combinations on long-term trends in population and community structure.
A

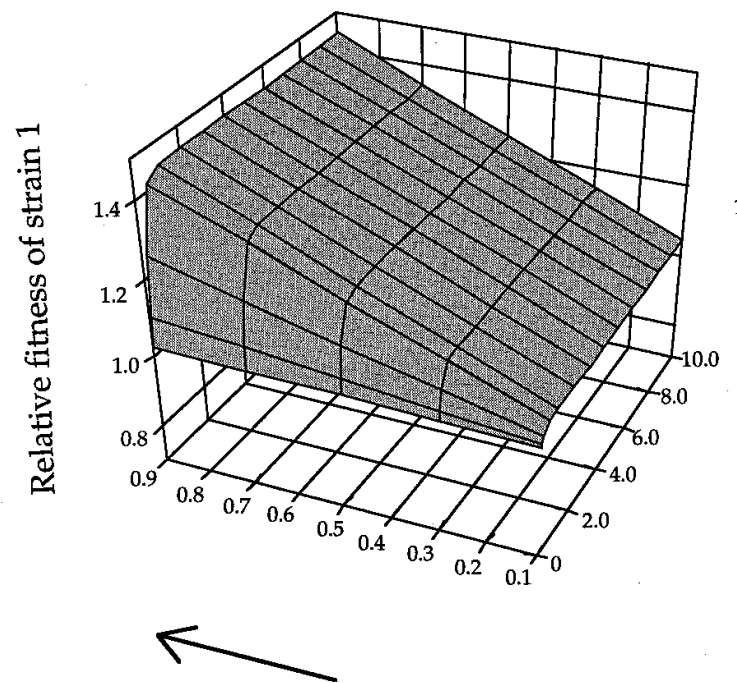

Frequency of strain 1
B

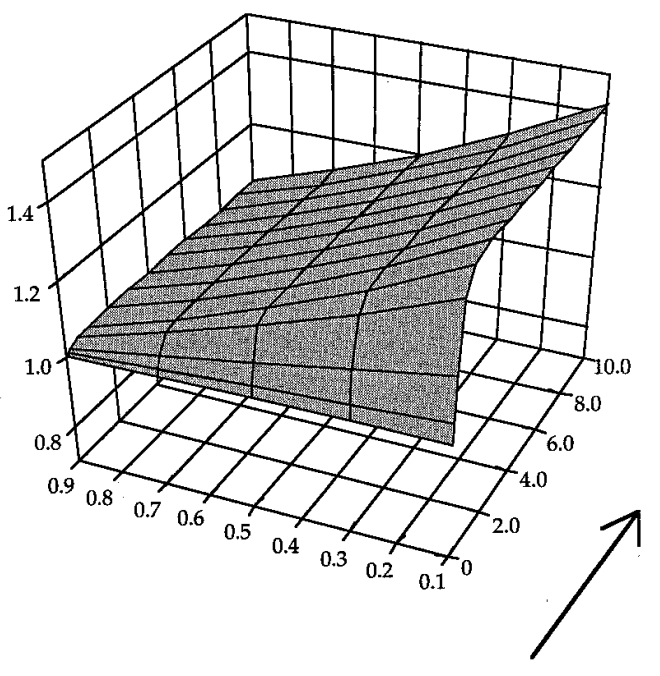

Total population density ( $x 10^{4}$ urediniospores)

Fig. 3. Calculated relationships among total population density (combined inoculum density) of two pathogen strains, frequency of strain 1 , and relative fitness of strain 1 when strains differ in their competitive ability for spore production. $\mathbf{A}, \beta_{1}=1.5$ and $\beta_{2}=1.0$; and $\mathbf{B}, \beta_{1}=1.0$ and $\beta_{2}=0.67$. Note that inter- and intrastrain competition effects are equal when $\beta=1$.0. Text has details of the calculations.

A

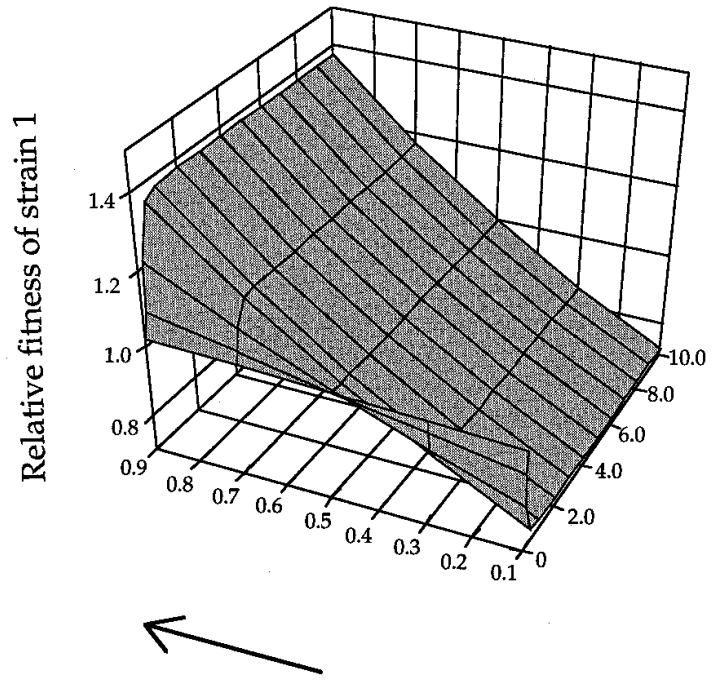

Frequency of strain 1
B

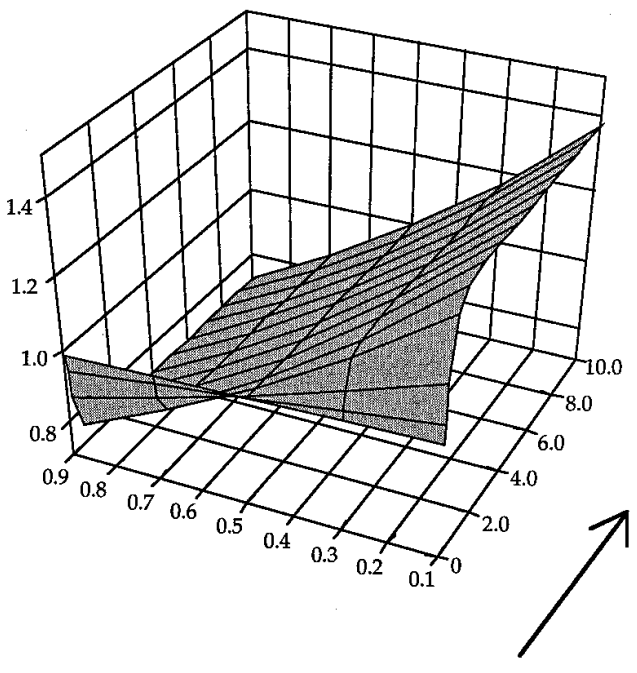

Total population density (x104 urediniospores)

Fig. 4. Calculated relationships among total population density (combined inoculum density) of two pathogen strains, frequency of strain 1 , and relative fitness of strain 1 when strains inhibit each other $\mathbf{A}$, more strongly $\left(\beta_{1}=\beta_{2}=1.5\right)$ or $\mathbf{B}$, more weakly $\left(\beta_{1}=\beta_{2}=0.67\right)$ than they inhibit themselves. Text has details of the calculations. 


\section{ACKNOWLEDGMENTS}

We thank the Department of Plant Pathology at the University of Minnesota and the USDA, ARS Cereal Rust Laboratory for providing the resources for this work. The research was conducted under project 22$18 \mathrm{H}$ with funding from the department's H. H. Flor Fellowship awarded to M. Newton. This manuscript was published as paper 191220005 of the contribution series of the Minnesota Agricultural Experiment Station. We thank J. Groth and C. Windels for strengthening the manuscript with their thoughtful comments.

\section{LITERATURE CITED}

1. Antonovics, J., and Fowler, N. L. 1985. Analysis of frequency and density effects on growth in mixtures of Salvia splendens and Linum grandiflorum using hexagonal fan designs. J. Ecol. 73:219-234.

2. Antonovics, J., and Kareiva, P. 1988. Frequency-dependent selection and competition: Empirical approaches. Philos. Trans. R. Soc. Lond. B Biol. Sci. 319:601-613.

3. Antonovics, J., and Levin, D. A. 1980. The ecological and genetic consequences of density-dependent regulation in plants. Annu. Rev. Ecol. Syst. 11:411-452.

4. Campbell, C. L., and Madden, L. V. 1990. Introduction to Plant Disease Epidemiology. John Wiley \& Sons, Inc., New York.

5. Clarke, B. 1972. Density dependent selection. Am. Nat. 106:1-13.

6. Davison, A. D., and Vaughan, E. K. 1964. Effect of urediospore concentration on determination of races of Uromyces phaseoli var. phaseoli. Phytopathology 54:336-338.

7. Durbin, R. D. 1984. Effects of rust on plant development in relation to translocation of inorganic and organic solutes. Pages 509-528 in: The Cereal Rusts. Vol. I: Origins, Specificity, Structure and Physiology. W. R. Bushnell and A. P. Roelfs, eds. Academic Press, Orlando, FL.

8. Futuyma, D. J. 1986. Evolutionary Biology, 2nd ed. Sinauer Associates, Inc., Sunderland, MA.

9. Gregory, P. H. 1948. The multiple infection transformation. Ann. Appl. Biol. 35:412-417.

10. Grime, J. P. 1979. Plant Strategies and Vegetation Processes. John Wiley $\&$ Sons, Chichester, England.

11. Hancock, J. G., and Huisman, O. C. 1981. Nutrient movement in hostpathogen systems. Annu. Rev. Phytopathol. 19:309-331.

12. Imhoff, M. W., Leonard, K. J., and Main, C. E. 1982. Patterns of bean rust lesion size increase and spore production. Phytopathology 72:441-446.

13. Irish, K. R. 1950. Studies on competition among physiologic races of the leaf rust of wheat. (Abstr.) Phytopathology 40:871-872.

14. Johnson, N. L., and Kotz, S. 1969. Discrete Distributions. Houghton Mifflin, Boston.

15. Kardin, M. K., and Groth, J. V. 1989. Density-dependent fitness interactions in the bean rust fungus. Phytopathology 79:409-412.

16. Katsuya, K., and Green, G. J. 1967. Reproductive potentials of races $15 B$ and 56 of wheat stem rust. Can. J. Bot. 45:1077-1091.

17. Kinkel, L. L., Newton, M. R., and Leonard, K. J. 1996. Quantifying microbial competition on leaves. Pages 265-276 in: Aerial Plant Surface Microbiology. C. E. Morris, P. C. Nicot, and C. Ngyuenthe, eds. Plenum Press, New York.

18. Leonard, K. J. 1969. Factors affecting rates of stem rust increase in mixed plantings of susceptible and resistant oat varieties. Phytopathology 59:1845-1850.

19. Leonard, K. J. 1969. Selection in heterogeneous populations of Puccinia graminis f. sp. avenae. Phytopathology 59:1851-1857.

20. Levin, B. R. 1988. Frequency-dependent selection in bacterial populations. Philos. Trans. R. Soc. Lond. B Biol. Sci. 319:459-472.

21. Lloyd, D. G. 1980. Benefits and handicaps of sexual reproduction. Evol. Biol. 13:69-111

22. Lotka, A. J. 1932. The growth of mixed populations: Two species competing for a common food supply. J. Wash. Acad. Sci. 22:461-469.

23. MacArthur, R. H., and Wilson, E. O. 1967. The Theory of Island Biogeography. Princeton University Press, Princeton, NJ.

24. Newton, M. R., Kinkel, L. L., and Leonard, K. J. 1996. Density- and frequency-dependent fitness in coexisting foliar pathogens. (Abstr.) Phytopathology (Suppl.) 86:S85.

25. Newton, M. R., Kinkel, L. L., and Leonard, K. J. 1997. Competition and density-dependent fitness in a plant parasitic fungus. Ecology 78:1774-1784.

26. Owera, S. A. P., Farrar, J. F., and Whitbread, R. 1983. Translocation from leaves of barley infected with brown rust. New Phytol. 94:111-123.

27. Petersen, L. J. 1959. Relations between inoculum density and infection of wheat by uredospores of Puccinia graminis var. tritici. Phytopathology 49:607-614.

28. Rai, B., and Upadhyay, R. S. 1983. Competitive saprophytic colonisation of pigeon-pea substrate by Fusarium udum in relation to environmental factors, chemical treatments and microbial antagonism. Soil Biol. Biochem. 15:187-191.

29. Roughgarden, J. 1979. Theory of Population Genetics and Evolutionary Ecology: An Introduction. Macmillan Publishing Co., Inc., New York.

30. Shaner, G. 1983. Growth of uredinia of Puccinia recondita in leaves of slow- and fast-rusting wheat cultivars. Phytopathology 73:931-935.

31. Sun, P., and Zeng, S. 1993. Discriminating synergism and antagonism of spores within species of phytopathogenic fungi. Phytopathology 83: 1051-1054.

32. Tilman, D. 1985. The resource ratio hypothesis of succession. Am. Nat. 125:827-852.

33. Volterra, V. 1931. Variations and fluctuations of the numbers of individuals in animal species living together. Pages 409-448 in: Animal Ecology. R. N. Chapman, ed. McGraw Hill, New York.)

34. Wallace, B. 1975. Hard and soft selection revisited. Evolution 29:465473.

35. Wardle, D. A., Parkinson, D., and Waller, J. E. 1993. Interspecific competitive interactions between pairs of fungal species in natural substrates. Oecologia 94:165-172.

36. Yang, X. B., and TeBeest, D. O. 1995. Competitiveness of mutant and wild-type isolates of Colletotrichum gloeosporioides $\mathrm{f}$. sp. aeschynomene on northern jointvetch. Phytopathology 85:705-710.

37. Yarwood, C. E. 1961. Uredospore production by Uromyces phaseoli. Phytopathology 51:22-27. 